\title{
How to Enhance the Threat Perceptions of Hazardous Chemicals for China's Urban Residents
}

Liu $\mathrm{Y}^{1 *}$ and Zhang $\mathbf{Q}^{2}$

${ }^{1}$ School of Management and Economics, Beijing Institute of Technology, China

${ }^{2}$ Hiwing Group of CASIC, China

${ }^{*}$ Corresponding author: Yongkui Liu, School of Management and Economics, Beijing

\section{Commentary}

Volume 3 Issue 3

Received Date: September 12, 2018

Published Date: September 28, 2018

Institute of Technology, No.5 South Street Zhongguancun Haidian District Beijing, China, Tel: +86-1326-0035-149; Email: liuyongkui_bit@163.com

\section{Abstract}

Risk communication of technological risk is tough in view of the limitation of the professional requirement for media and knowledge requirement for the individual. This research focuses on the communication dilemma in the field of hazardous chemicals from the viewpoint of relationships between the communication channel and risk perceptions, expounds the characteristics of different communication channels, and provides theoretical support for Chinese urban residents to select appropriate channels to receive hazardous chemicals information and enhance their threat perception.

Keywords: Risk; Channel Preference; Risk Perception; Hazardous Chemicals

\section{Commentary}

Recently, China is in a period of accelerated urbanization, and a large number of wealth and population continue to gather in the city. However, it should not be overlooked that since the reform and development, various dangerous chemicals left with the urban construction and development increasingly become the source of threatening urban safety [1]. For example, "11.22" oil pipeline leakage and explosion accident in Qingdao City, and "8.12" hazardous chemicals warehouse fire and explosion accident in Tianjin Port which cause a large number of casualties have shown this phenomenon. In fact, urban residents, as laymen, generally rely on intuition to judge the risk of hazardous chemicals, while extreme technical complexity and authoritative department information monopoly of hazardous chemicals cause people in danger without knowing it, or cause people to make radical action under the pressure of public opinion. There are two reasons for above phenomenon: on the one hand, a large number of knowledge reserve is required for the public to understand the threat of hazardous chemicals, while the public are lack of enthusiasm to understand the relevant knowledge in current fragmented information age; on the other hand, very few journalists have any specific expertise in hazardous chemicals, it is difficult for media columns and authors to own sufficient knowledge to make accurate reports, while some medium cannot provide enough space or capacity to carry these knowledge [2]. Therefore, the threat information of hazardous chemicals spread by current media is not accurate enough, which cannot effectively improve the risk perception of urban residents; and urban residents are also lack of the relevant knowledge for the threat of hazardous chemicals, it is in urgent need of appropriate 
media channels to guide them to better obtain and improve the knowledge of hazardous chemicals. It can be seen that, based on the spread characteristics of media channels, the improvement of risk perception of hazardous chemicals for urban residents, has become an important issue related to the government credibility and social stability.

Relevant research found that, firstly, faced with technical complexity and stigma characteristics of

Hazardous chemicals, the reaction of mass media is less responsive: on the one hand, the information of newspaper, television and other mass medium is relatively lagged behind, and it cannot appear in the public view until passing layers of examination and approval [3]. Furthermore, the threat information of hazardous chemicals spread by mass media is based on the mode of "you speak, I listen; you write, I read", which is lack of interactivity [4]. On the other hand, the limited capacity has limited its spread efficiency, especially no comprehensive report lead to a large number of terms or professional terminology which cannot be understood by general residents are mixed in the information, and people can only passively absorb and cannot make active learning.

Secondly, the network is more and more developed today, and using network platform to obtain information has become increasingly common, so the role of social media in spreading the threat information of hazardous chemicals is increasingly stronger. However, lack of gatekeeper also leads to the disadvantage of poor authority for social media. Open network allows people to freely release information, including a large number of false information, reducing the trust of people in social media [5].

Thirdly, expert explanation, theoretical lecture and other face-to-face communication forms have a more intuitive understanding, and its interactive effect is also easy to be accepted by urban residents, so its effect is also better than that of mass media. But the urban residents are influenced under social pressure when people choose face-to-face communication [6,7]. On the one hand, hazard information transmitted by face-to-face communication has integrated the orientation of managers and social mainstream public opinion, with the characteristic of incomplete content coverage. On the other hand, the transmission form of face-to-face communication does not benefit from the active absorption of audience, especially in training seminars, many audiences attend under pressure, and if the contents of training seminars cannot be effectively mastered, and then communication effect will be affected.
The research of risk perception is relatively rich, which relates to disasters of flood, chemical leakage, nuclear radiation, food safety [8-11]. Slovic considers that hazardous chemicals have some characteristics of high fear, medium unknown, uncontrollability, devastating, impact directly, involuntariness and affect oneself [12]. In recent years, some domestic scholars have begun to proceed with communication channel, and pay attention to the risk perception of hazardous chemicals. For example, Xie, et al. have proposed that TV can arouse higher risk perception than that of web page after comparing the influences of two communication channels on risk perception; While Xue, et al. and Ren mainly concern about the influences of micro-blog, social media and other emerging medium on the risk perception of PX project [13-15]. The relevant researches of foreign scholars focus on the analysis of channel types. For example, Lindell and Perry, find that warning information can be transmitted through face-to-face contact, telephone, alarm, mobile speaker, radio and other channels; Hance, et al. and Mileti, et al. believe that the main risk communication channel for the community is electronic media; Perry and Nelson argue that, subject to personal preference, community needs to carry out crosschannel risk communication $[16,20]$. So, for the issue of accident disaster risk perception with technical complexity characteristics, the relevant researches are lack of depth study on the complex relationship between communication channel and risk perception, without solving the issue of cross-channel communication. Thus it can be seen that, hazard information released by different communication channels will cause different degrees of risk perception, and it has a high theoretical value to analyze the influence of channel preference and channel combination on risk perception of hazardous chemicals.

\section{Acknowledgement}

This paper is sponsored by National Social Science Funds (Grant No. 16BGL175) of "Mechanism and path of risk communication responding to dangerous chemical hazards for urban residents". All the authors would like to express our sincere gratitude to the sponsor.

\section{References}

1. Benekos I, Diamantidis D (2017) On risk assessment and risk acceptance of dangerous goods transportation through road tunnels in Greece. Saf Sci 91: 1-10. 
2. David A (2006) Globalization of Disaster: Trends, Problems AND Dilemmas. Journal of International Affairs 59(2): 1-22.

3. Song GY (2016) New media how to effectively draw on the advantages of traditional media. Young Journalists (9): 102-013.

4. Xiao L, Xu SH, Wang Q (2016) Social media development and research review. Research on Library Science (14): 13-16.

5. Zhao PP (2016) Analysis of the Spread of Internet Rumors in the Social Media Era. Chinese Culture Forum (9): 153-155.

6. Zuo YZ (2009) On the choice of contemporary Chinese youth under social pressure. Journal of Fujian Provincial Committee Party School (3): 76-80.

7. Peng SC, Yang AM, Cao LH, Yu S, Xie DQ (2017) Social influence modeling using information theory in mobile social networks. Information Sciences 379(10): 146-159.

8. Lindell MK, Mumpower JL, Huang SK, (2017) Perceptions of Protective Actions for a Water Contamination Emergency. Journal of Risk Research 20(7): 887-908

9. Heath RL, Lee J, Palenchar MJ (2018) Risk Communication Emergency Response Preparedness: Contextual Assessment of the Protective Action Decision Model. Risk Analysis, 38(2): 333-344.

10. Vyncke B, Perko T, Van GB (2017) Information Sources as Explanatory Variables for the Belgian Health-Related Risk Perception of the Fukushima Nuclear Accident. Risk Analysis 37(3): 570-582.

11. Rossi MSC, Stedefeldt E, da Cunha DT, Rosso VV (2017) Food Safety Knowledge, Optimistic Bias and
Risk Perception among Food Handlers in Institutional Food Services. Food Control 73(1): 681-688.

12. Slovic P (2016) The Perception of Risk. Routledge.

13. Xie XF, Li J, Yu QY (2008) How Can a Risk Be Increased? An analysis of risk communication channels. Acta Psychologica Sinica 40(4): 456-465.

14. Xue K, Wang LL, Yu MY (2015) Study on the Impact of Social Media on Audience Risk Perception: A Case Study of PX Project Explosion in Gulei Peninsula. Journalism Bimonthly (6): 101-107.

15. Ren JH (2016) Spread and reflection of microblogging information in emergencies - An empirical analysis based on the "PX Project Explosion in Zhangzhou, Fujian Province”. Social Scientist (8): 5355.

16. Lindell MK, Perry RW (1987) Warning mechanisms in emergency response systems. International Journal of Mass Emergencies and Disasters 5: 137-153.

17. Lindell MK, Perry RW (1992) Behavioral foundations of community emergency planning. Washington, DC: Hemisphere.

18. Hance B, Chess C, Sandman P (1988) Improving dialogue with communities. New Brunswick: New Jersey Department of Environmental Protection pp: 34.

19. Mileti DS, Sorensen JH, O’Brien PW (1992) Toward an explanation of mass care shelter use in evacuations. International Journal of Mass Emergencies and Disasters 10(1): 25-42.

20. Perry RW, Nelson L (1991) Ethnicity and hazard information dissemination. Environmental Management 15(4): 581-587. 